\title{
DEFICIENCIA AUDITIVA: AUTOCONCEPTO, AUTOESTIMA Y SÍNTOMAS PSICOPATOLÓGICOS
}

\author{
José Ignacio Pérez
}

Maite Garaigordobil

Departamento de Personalidad, Evaluación y Tratamientos Psicológicos. Facultad de Psicologla. Universidad del Pais Vasco

\section{RESUMEN}

El estudio se plantea 3 objetivos: 1) analizar comparativamente el autoconcepto, la autoestima y sintomas psicopatológicos en personas con y sin deficiencia auditiva; 2) explorar diferencias de género en el autoconcepto, la autoestima y síntomas psicopatológicos en ambas muestras; y 3) estudiar las relaciones existentes entre el autoconcepto y la autoestima con sintomas psicopatológicos en personas con y sin deficiencia auditiva. La muestra está configurada por 130 participantes de 19 a 40 años, 72 sin deficiencia y 58 con deficiencia auditiva. El estudio empleó una metodología descriptiva y correlacional. Se aplicaron 3 instrumentos de evaluación: LAEA, EA, SCL-90-R. Los ANOVAs confirmaron que los participantes con deficiencia auditiva no tenian diferencias en autoconcepto $y$ autoestima respecto a los norm-oyentes, sin embargo tenian puntuaciones

\footnotetext{
Correspondencia: Dpto. de Personalidad, Evaluación y Tratemientos Psicológicos.Facultad de Psicologla. Universidad del Pais Vasco. Avda. de Tolosa 70. 20018 Donostia-San Sebastián. Tfno: 943 - 015641 / Fax: 943 - 015670 E-mail: joseignacio.perez@ehu.es.

Maite Garaigordobil y José Ignacio Pérez son profesores en el Dpto. de Personalidad, Evaluación y Tratamientos Psicológicos, en la Facultad de Psicologla de la Universidad del Pals Vasco. El estudio que se expone ha sido financiado por el Vicerrectorado de Investigación de la Universidad del Pals Vasco (1/UPV 00006.231-H-15910/2004). Correspondencia relacionada con el artlculo puede ser enviada a Jose lgnacio Pérez, Facultad de Psicologla. Universidad del Pals Vasco, Avenida de Tolosa 70, 20018 DonostiaSan Sebastián. E-mail: Joseignacio.perez@ehu.es http://www.sc.ehu.es/joseignacio
} 
superiores en todos los sintomas psicopatológicos excepto en somatización. No se evidenciaron diferencias de género en autoconcepto, autoestima, y sintomas psicopatológicos ni en los participantes con deficiencla auditiva ni en los normoyentes: ansiedad fóbica. Los coeficientes de Pearson pusieron de relieve correlaciones negativas del autoconcepto y la autoestima con la puntuación total de sintomas psicopatológicos.

\section{Palabras clave: DEFICIENCIA AUDITIVA, AUTOCONCEPTO, AUTOESTIMA, PSICOPATOLOGIA.}

\section{SUMMARY}

The purpose of this study is three-fold: 1) to analyze the selfconcept, self-esteem and psychopathological symptoms in subjects with and without hearing impaiment; 2) to explore whether there were differences according to gender in the self-concept, self-esteem and psychopathological symptoms in both samples; 3) to study the relationships between self-concept/self-esteem and psychopathological symptoms in particlpants with and without hearing impairment. The sample is made up of 130 participants aged 19 to 40, 72 without hearing impairment and 58 with hearing impairment. The study uses a descriptive and correlational methodology. For the measurement of the variables three assessment instruments were applied: LAEA, EA, SCL-90-R. The ANOVA results show that both samples did not have any significant differences in self-concept and self-esteem. However, hearing impaired had significantly higher scores In all psychopathological symptoms except in somatization. Both samples did not show significant gender differences neither in self-concept/self-esteem nor in psychopathological symptoms: impaired women only had higher scores than men in the distress index, and non impaired women in the distress index and phobic anxiety. Pearson coefficients showed significant inverse relationships between selfconcept/self-esteem and total psychopathological symptoms.

Key words: HEARING IMPAIRMENT, SELF-CONCEPT, SELF-ESTEEM, PSYCHOPATHOLOGY. 


\section{INTRODUCCIÓN}

\section{Deficlencla auditlva: conceptuallzacion}

La deficiencia auditiva, hipoacusia o sordera consiste en la disminución o pérdida de la audición. Esta definición no determina las características de los sujetos incluidos dentro de este grupo, puesto que los efectos de la deficiencia auditiva son múltiples y las diferencias individuales muy grandes: pueden producirse alteraciones de distinto grado en el desarrollo del lenguaje, el habla y la voz, así como en el desarrollo cognitivo y de las habilidades sociales. Las variables fundamentales que determInan el tipo de deficiencia auditiva son: 1) el momento en el que se produce la pérdida auditíva, lo que permite diferenciar entre deficiencias preiocutivas (congenitas o acaecidas durante los tres primeros años de vida) y postiocutivas; 2 ) el grado de pérdida auditiva, según el cual se distingue entre hipoacusia leve o ligera (la pérdida se sitúa entre los 20-40 db), media o moderada (40-70 db), severa (70-90 db), profunda (pérdidas superiores a los 90 db), y totai o anacusia (ausencia de resto auditivo); 3 ) la estructura fisiológica afectada, atendiendo a lo cual se diferencia entre hipoacusias de transm/slón o conductivas (localizadas en el oldo externo y/o medio) y neurosensoriales (localizadas en la cóclea o en el nervio auditivo); 4) el tiempo transcurrido entre el momento en el que sobreviene la pérdida auditiva y la detección de la misma; y 5) la premura con la que se implemente una intervención que de respuesta a las necesidades espec/ficas derivadas de la deficiencia auditiva mediante un equipamiento adecuado (por ejemplo, audifonos o implantes cocleares), estimulaclón temprana, rehabilitación logopédica, sistemas aurnentativos y/o alternativos de comunicación, etc. (Santana y Torres, 2003). SIn embargo, conviene no olvidar que además de las varlables que definen el tipo de deficiencla auditiva, existen otras muchos factores diferenciales que también dan razón de la amplia variabilidad interindividual, tales como el hecho de que los padres sean sordos u oyentes, la aceptación de la deficiencia auditiva de su hijo/a, la utilización a nivel familiar y educativo de un determinado sistema de comunicación (oral, bimodal, palabra complementada, lengua de signos), o la modalidad de escolanización, entre otros. 
Los términos deficiencia auditiva, hipoacusia y sordera por lo general se consideran sinónimos en la literatura sobre el tema $-y$ como tal se utilizan en el presente trabajo-, pese a que desde perspectivas educativas o socioculturales se establezcan en ocasiones diferencias conceptuales entre ellas. Así, en el ámbito de la educación a veces se utiliza el término hipoacúsico para referirse a aquel sujeto cuya audicion, pese a ser deficiente, resulta funcional para la vida ordinaria y permite la adquisición del lenguaje oral-con prótesis o sin ella- por via auditiva, y se emplea el término sordo cuando la audición no es funcional para la vida cotidiana y no posibilita la adquisición del lenguaje oral por vla auditiva (aunque si pueda lograrse, en mayor o menor grado, por vla visual). Desde una perspectiva sociocultural, cabe señalar que en los últimos años investigadores y grupos de sordos han revindicado el derecho a considerar a la «comunidad sorda» como un grupo cultural con entidad propia. Desde esta perspectiva distinguen entre el término «sordo», aplicable a todo aquel que presenta algún tipo de pérdida auditiva por el mero hecho de tenerla, y «Sordo», utilizado únicamente para referirse a aquel que, con relativa independencia de su pérdida auditiva, se siente parte de la comunidad sorda, conoce su lenguaje y comparte sus peculianidades culturales (relacionadas fundamentalmente con el papel predominante de la experiencia visual para este colectivo). Por consiguiente, desde esta última perspectiva se resalta la importancia de la identificación con el grupo social de referencia, lo que lleva a muchos adultos sordos a subrayar la necesidad de que se reconsidere la noción de deficiencia auditiva, ya que sostienen que cuando se comunican entre si en lengua de signos no se sienten deficitarios, sino simplemente diferentes. A pesar de que el debate en torno a si los sordos pueden o no ser considerados una minorla cultural propiamente dicha continúa abierto, lo cierto es que cada vez son más quienes apoyan una perspectiva sociocultural de la sordera (Jambor y Elliott, 2005).

Autoconcepto, autoestima, síntomas psicopatológicos y deficiencia auditiva

En los últimos años la investigación del autoconcepto y la autoestima está cobrando gran relevancia en el contexto de la identificación de 
factores protectores de problemas psicopatológicos. Su importancia en relación con aspectos tales como el bienestar personal, la salud mental, el éxito profesional o las relaciones sociales -entre otroshan atraído el interés de muchos investigadores de las ciencias humanas y sociales. El autoconcepto y la autoestima resultan variables críticas en el estudio de las deficiencias sensoriales (Satapathy y Singhal, 2001). Durante el proceso de construcción del concepto de uno mismo, la interacción con las personas próximas juega un papel destacado, con lo que las persona con algún tipo de déficit sensorial puede incorporar a su propia imagen, en mayor o menor medida, algunos de los aspectos o estereotipos que se le atribuyen. Además, la comparación social desempeña un papel fundamental en el desarrollo de la autoestima, puesto que la persona se evalúa en comparación con los otros; en este sentido, cualquier información negativa que se derive de este proceso en base a limitaciones significativas en el funcionamiento de alguna de las habilidades adaptativas supone una amenaza potencial para la autoestima y un riesgo para la salud, puesto que diversos estudios han evidenciado la relación entre comparación social negativa y problemas psicológicos (Allen y Gilbert, 1995; Swallow y Kuiper, 1988).

Al hilo de este argumento cabría esperar, por tanto, que los jóvenes y adultos con deficiencia auditiva evidenciaran bajos niveles de autoconcepto y autoestima, ya que pertenecen a una minoría caracterizada por una deficiencia que parece probable que internalice ciertas actitudes negativas - 0 cuando menos «benévolas"-del grupo mayoritario normoyente (Lane, 1992). Sin embargo, en su conjunto, las escasas investigaciones empíricas llevadas a cabo sobre el autoconcepto y la autoestima de jóvenes y adultos con deficiencia auditiva arrojan resultados contradictorios. Si bien es cierto que los datos de algunos estudios evidenciar en los jóvenes y adultos sordos niveles significativamente más bajos que la población general en autoconcepto (Weisel y Kamara, 2005) y autoestima (Bat-Chava, 1994; Mulcahy, 2002; Schlesinger, 2000), los resultados de otras investigaciones no confirman que los participantes con deficiencias auditivas muestren necesariamente un autoncocepto-autoestima más bajos que los sujetos normoyentes (Bat-Chava, 2000; Crowe, 2003; Emerton, 1996). 
Por otro lado, la prevalencia de sintomatología psicopatológica entre los jóvenes y adultos con deficiencia auditiva ha sido ampliamente estudiada, y la literatura sobre el tema evidencia que los jóvenes y adultos con deficiencia auditiva frecuentemente presentan puntuaciones más altas en síntomas psicopatológicos en comparación con sujetos normoyentes, especialmente sintomas ansiosos (De Graaf y Bljl, 2002; Fellinger et al., 2005; Tambs, 2004) y depresivos (De Graaf y Bijl, 2002; Mulcahy, 2002; Tambs, 2004).

Autoconcepto, Autoestima y síntomas psicopatológicos: Dlferenclas de género

Los resultados de los estudios que han analizado las diferencias de género en el autoconcepto y la autoestima de jóvenes y adultos con sordera no muestran diferencias significativas en función del género (Crowe, 2003), en la linea de otros trabajos que tampoco las han encontrado en la población general (Garalgordobil, Durá, y Pérez, 2005; Lameiras y Rodríguez, 2003; Matud, Ibánez, Marrero, y Carballeira, 2003).

En lo que a sintomas psicopatológicos se refiere, la literatura sobre la deficiencia auditiva evidencia diferencias significativas en función del género, con mayores niveles generales de sintomatología en mujeres que en hombres (De Graaf y Bijl, 2002; Fellinger et al., 2005), en la misma línea que la población normoyente, en la que las mujeres parecen mostrar mayores niveles psicopatológicos tanto en los índices globales como en alguna de las dimensiones del SCL90. En concreto, en la población general española algunos estudios (González de Rivera, De las Cuevas, Rodrlguez Abuín, y Rodriguez Pulido, 2002) han hallado en las mujeres puntuaciones superiores en las dimensiones de somatización, depresión, ansiedad, ansiedad fóbica, asl como en el indice sintomático general, en el total de sintomas positivos, y en el índice de distrés por los síntomas.

Relaciones entre autoconcepto-autoestima y psicopatología

Los estudios que han analizado las conexiones del autoconceptoautoestima con síntomas psicopatológicos tanto con sujetos 
deficientes auditivos (De Graaf y Bijl, 2002; Satapathy y Singhal, 2001) como en la población general (Fan y Fu, 2001; Montt y Chavez, 1996) muestran relaciones inversas entre ambas variables, evidenciando correlaciones positivas entre autoconcepto, autoestima y salud mental.

\section{Objetivos a hipótesis del estudlo}

El estudio se plantea 3 objetivos: 1) analizar comparativamente el autoconcepto, la autoestima y diversos síntomas psicopatológicos en personas con y sin deficiencia auditiva; 2) explorar la existencia de diferencias de género en el autoconcepto, la autoestima y síntomas psicopatológicos en personas con y sin deficiencia auditiva; $y 3$ ) estudiar las relaciones existentes entre el autoconcepto y la autoestima con síntomas psicopatológicos en personas con y sin deficiencia auditiva. La investigación propone 5 hipótesis: 1) Las personas con deficiencia auditiva tendrán puntuaciones en autoconcepto y autoestima significativamente inferiores a los normoyentes; 2) Las personas con deficiencia auditiva tendrán puntuaciones significativamente superiores en sintomas psicopatológicos respecto a las normoyentes; 3) No se encontrarán diferencias de género en el autoconcepto y la autoestima de los participantes con y sin deficiencia auditiva; 4) Las mujeres con y sin deficiencia auditiva tendrán puntuaciones superiores en síntomas psicopatologicos; y 5) Los participantes con alto autoconcepto y autoestima tendrán pocos sintomas psicopatológicos.

\section{METODOLOGÍA}

\section{Participantes}

La muestra está configurada por 130 participantes de 19 a 40 años de edad, pertenecientes a las tres provincias de la Comunidad Autónoma Vasca. La muestra incluye una submuestra de 72 participantes sin deficiencia auditiva y otra submuestra de 58 participantes con deficiencia auditiva.

La muestra de personas con deficiencia auditiva son usuarios de la Asociación de Personas Sordas de Bilbao y Vizcaya y la Unión de Sordos de Guipúzcoa que voluntariamente se ofrecieron a participar 
en el estudio. En ambos casos se trata de asociaciones cuyo objetivo es crear y fomentar la unidad entre las personas sordas, para promover su participación en actividades normativas, culturales, sociales y recreativas, a fin de conseguir asi su plena integración social y laboral. De los 58 participantes, el $56.9 \%$ son varones (33) y el $43.1 \%$ mujeres (25). La muestra incluye sujetos con distintos niveles de estudios: estudios primarios $(25.9 \%)$, secundarios $(1.7 \%)$, y FP o Bachiller (70.7\%). El $20.7 \%$ son estudiantes, el $55.2 \%$ son trabajadores manuales, el $8.6 \%$ realiza trabajos profesionales que implican tareas intelectuales, el $13.8 \%$ está en paro, y $1.7 \%$ jubilado. En relación al nivel de pérdida auditiva de los participantes del estudio, el $8.6 \%$ tiene un grado de pérdida ligera (20-40), el $27.6 \%$ un nivel de pérdida media (40-70), el $12.1 \%$ pérdida severa (70-90), el $17.2 \%$ pérdida profunda (más de 90 ), el $15.5 \%$ tiene un grado de pérdida total o anacusia, en tanto que el $19 \%$ no conoce su grado de pérdida (si bien son usuarios habituales de la lengua de signos). Sobre el momento evolutivo en el que se produjo la deficiencia auditiva, el $93.1 \%$ presentan una pérdida prelocutiva (congénita o sobrevenida durante los 3 primeros años de edad). En cuanto a los antecedentes familiares, el $87.9 \%$ tiene padres oyentes, un $5.2 \%$ tiene padres sordos, el $1.7 \%$ tiene madre sorda/ padre oyente, mientras que el $3.4 \%$ tiene madre oyente/padre sordo, y el $1.7 \%$ tiene padres oyentes y un hermano sordo. En lo que se refiere a la educación, el $50 \%(29)$ ha tenido una educación oralista, el $6.9 \%$ (4) educación con lengua de signos, el $10.3 \%$ (6) educación bimodal, el $1.7 \%$ (1) ha tenido en primaria y secundaria una educación oralista, $y$ en bachillerato una lengua de signos, $y$ el $31 \%$ (18) ha tenido una educación bilingule. Además, el $93.1 \%(54)$ es usuario habitual de la lengua de signos, mientras que el $5.2 \%$ (3) no la utiliza.

La selección de la muestra de personas sin deficiencia auditiva fue aleatoria tomando como referencia los censos de las tres capitales de las provincias de Comunidad Autónoma Vasca. Teniendo en cuenta diversos estratos: indice de población en cada ciudad, sexo, nivel de estudios, ocupación laboral... se emplé un muestreo aleatorio simple. De los 72 participantes sin deficiencia auditiva el $55.6 \%$ son varones (40) mientras que el $44.4 \%$ son mujeres (32). Teniendo en cuenta la necesidad de cierto nivel de homogeneidad en estudios y ocupación laboral entre ambas submuestras, la muestra de población normoyente 
contiene sujetos con distintos niveles de estudios: estudios primarios $(12.5 \%)$, secundarios $(16.7 \%)$, y FP - Bachiller $(70.8 \%)$. Además, los participantes tienen distintos niveles de ocupación: estudiantes $(29.2 \%)$, trabajadores manuales $(54.2 \%)$, profesionales y trabajadores de actividades intelectuales $(12.5 \%)$, y parados $(4.2 \%)$.

\section{instrumentos de Evaluación}

Con la finalidad de medir las variables dependientes se administraron 3 instrumentos de evaluación con adecuadas garantías psicométricas de fiabilidad y validez.

LAEA. Listado de adjetivos para la evaluación del autoconcepto en adolescentes y adultos (Garaigordobil, en prensa).

El listado está compuesto por 57 adjetivos (confiada, cooperativa, inteligente, feliz, sensible) y se solicita al sujeto que valore en una escala de estimación de 0 a 4 (nada-mucho) en qué grado estos adjetivos le definen o describen su personalidad. Un estudio realizado con una muestra de 634 sujetos obtuvo un coeficiente alpha de Cronbach de .92, y un coeficiente de Spearman-Brown de .83. La fiabilidad test-retest obtenida con una muestra de 142 estudiantes universitarios y un intervalo de 40 dias fue alta $(r=.83, p<.001)$, evidenciando la estabilidad temporal de la prueba. Para analizar la validez del LAEA, se realizaron correlaciones con otros instrumentos que miden el autoconcepto (AF-5, Garcla y Musitu, 1999) y con una escala de autoestima (EA, Rosenberg. 1965), obteniéndose correlaciones significativas del LAEA con el AF-5 $(r=.71, p<.001)$ y con la EA $(r=.63, p<.001)$ que muestran validez de constructo.

$E A$. Escala de autoestima (Rosenberg, 1965). Evalúa la autoestima general con 10 afirmaciones que aluden a sentimientos globales de autovaloración (en general estoy satisfecho conmigo mismo), 5 de los cuales están redactados en sentido positivo y 5 en sentido negativo. El sujeto debe leer las afirmaciones e informar en qué medida pueden ser aplicadas a él mismo, haciendo la valoración sobre una escala de tipo Likert con 4 categorlas de respuesta (de muy de acuerdo a muy en desacuerdo). La fiabilidad de la prueba ha sido ampliamente documentada en la literatura. McCarthy y Hoge (1982) han informado de coeficientes de consistencia (alpha de Cronbach) que se sitúan 
entre .74 y .77 , y de fiabilidad test-retest de .63 (intervalo de 7 meses) y de .85 (intervalo de 2 semanas). La validez de la escala como medida unidimensional de la autoestima ha sido también comprobada en varios estudios (Rosenberg, 1965; Silber y Tippett, 1965).

SCL-90-R. Cuestionario de 90 sintomas revisado (Derogatis, 1983/ 2002). Compuesto por 90 Items distribuidos en 10 escalas psicopatológicas: somatización (vivencias de disfunción corporal, con alteraciones neurovegetativas de los sistemas cardiovascular, respiratorio, gastrointestinal y muscular), obsesión-compulsión (conductas, pensamientos e impulsos que el sujeto considera absurdos e indeseados, que generan intensa angustia y que son dificiles de resistir, evitar o eliminar), sensibilidad interpersonal (sentimientos de timidez y vergüenza, tendencia a sentirse inferior a los demás, hipersensibilidad a las opiniones y actitudes ajenas $y$, en general, incomodidad e inhibición en las relaciones interpersonales), depresión (vivencias disfóricas, anhedonia, desesperanza, impotencia y falta de energia, ideas autodestructivas y otras manifestaciones cognitivas y somáticas características de los estados depresivos), ansiedad (manifestaciones clinicas de la ansiedad, tanto generalizada como aguda o "pánico», incluye signos generales de tensión emocional y sus manifestaciones psicosomáticas), hostilidad (pensamientos, sentirnientos y conductas propios de estados de agresividad, ira, irritabilidad, rabia y resentimiento), ansiedad fóbica (miedo persistente, irracional y desproporcionado a un animal o persona, lugar, objeto o situación, generalmente complicado por conductas de evitación o de huida, con un mayor peso en la escala de los sintomas de agorafobia y fobia social que los de la fobia simple), ideación paranoide (conducta paranoide, que incluye suspicacia, centralismo autorreferencial e ideación delirante, hostilidad, grandiosidad, miedo a la pérdida de autonomla y necesidad de control), psicoticismo (espectro psicótico que se extiende desde la esquizoidia leve hasta la psicosis florida, y que en la población general se relaciona más con sentimientos de alienación social que con psicosis clinicamente manifiesta), y escala adicional (síntomas misceláneos que constituyen un claro referente de depresión melancólica). Del sumatorio de las puntuaciones de las 10 escalas se obtiene una puntuación total en el SCL-90-R. Además la prueba permite calcular el Indice sintomático general (GSI), medida 
generalizada e indiscriminada de la intensidad del sufrimiento psiquico y psicosomático global, el total de sintomas positivos (PST), número de sintomas presentes, y el Indice de distrés de sintomas positivos (PSDI), que relaciona el sufrimiento o «distrés» global con el número de sintomas. Resultados de estudios con muestra española (González de Rivera et al., 2002) sugieren buena fiabllidad de la prueba, siendo coherentes con los del autor (Derogatis, 1983/2002). Los coeficientes alpha oscilan entre .81 y .90 . La estabilidad temporal (entre .78 y .90 ) con un intervalo test-retest de una semana muestra estabilidad de las puntuaciones a lo largo del tiempo. Otros estudios de validez muestran la relación entre el perfil de las dimensiones sintomáticas y el grupo diagnóstico al que pertenece la muestra clínica, encontrando puntuaciones significativamente mayores en las muestras psiquiátricas que en las muestras no clinicas (De las Cuevas y González de Rivera, 1991). Los estudios originales del autor con muestras americanas evidencian validez de constructo (Derogatis y Cleary, 1977) y convergente dadas las altas correlaciones de las dímensiones sintomáticas con el MMPI en pacientes psiquiátricos (Derogatis, Rickels y Rock, 1976), asi como validez de criterio o empírica (Derogatis, 1983/2002).

\section{Diseño y procedimiento}

El estudio realizado emplea una metodologla descriptiva y correlacional. Para evaluar las variables objeto de estudio se administran tres instrumentos de evaluación: La escala de autoestima (EA, Rosenberg, 1965), el Listado de adjetivos para la evaluación del autoconcepto en adolescentes y adultos (LAEA) (Garaigordobil, en prensa), y el Cuestionario de 90 síntomas revisado (SCL-90-R) (Derogatis, 1983/2002). La administración de los instrumentos de evaluación a los 25 sujetos de Guipúzcoa la llevó a cabo la trabajadora social de la asociación (ella misma sorda) y una intérprete de Lengua de Signos Española (L.S.E.) de la propia asociación. En el caso de las 33 personas de Vizcaya, para la cumplimentación de los cuestionarios de evaluación se contó con la ayuda de una estudiante de ciclo superior de psicologla con un hermano sordo y un conocimiento fluido de L.S.E., y la propia intérprete de L.S.E. de la asociación. Los cuestionarios se pasaron individualmente o en pequeños grupos de no más de 3 
personas. La metodología variaba en función de las dificultades de comprensión lectora. En aquellos casos en los que la comprensión lectora era buena, las responsables de la evaluación se limitaban a solventar individualmente las dudas puntuales que pudieran surgir, utilizando para ello la L.S.E. En los casos de problemas de comprensión lectora más graves, las intérpretes traducian directamente los ítems a L.S.E. y aclaraban cualquier duda de forma signada para facilitar la cumplimentación de los cuestionarios. A los participantes sin deficiencia auditiva la batería de evaluación fue aplicada por psicologos y estudiantes de psicologla formados en seminarios con esta finalidad. La aplicación de las pruebas se llevó a cabo en 2 sesiones de evaluación.

\section{RESULTADOS}

En primer lugar, se comprobó mediante un ANOVA si existian diferencias significativas en el autoconcepto, la autoestima y los sintomas psicopatológicos de los participantes con deficiencia auditiva en función del grado de pérdida auditiva, no encontrándose diferencias en ninguna de las variables medidas: autoconcepto, $F(4,42)=.44, p>$ .05 , autoestima, $F(4,42)=.42, p>.05$, somatización, $F(4,42)=.83, p$ $>.05$, obsesión-compulsión, $F(4,42)=.25, p>.05$, sensibilidad interpersonal, $F(4,42)=.15, p>.05$, depresión, $F(4,42)=.39, p>.05$, ansiedad, $F(4,42)=1.12, p>.05$, hostilidad, $F(4,42)=.48, p>.05$, ansiedad fóbica, $F(4,42)=.30, p>.05$, ideación paranoide, $F(4,42)=$ $1.12, p>.05$, psicoticismo, $F(4,42)=.71, p>.05$, escala adicional, $F$ $(4,42)=1.01, p>.05$, total de las escalas del SCL-90-R, $F(4,42)=.48$, $p>.05$, indice sintomático general, $F(4,42)=.48, p>.05$, total de sintomas positivos, $F(4,42)=1.22, p>.05$, e indice de distrés de sintomas positivos, $F(4,42)=.38, p>.05$. Debido a estos resultados, el resto de los análisis estadísticos se llevaron a cabo sin establecer diferencias en función del grado de pérdida auditiva.

Autoconcepto, autoestima y síntomas psicopatológicos en sujetos con y sin deficlencla auditiva

Con la finalidad de comparar el autoconcepto, la autoestima y diversos sintomas psicopatológicos en personas con y sin deficiencia 
auditiva (ambos sin trastornos mentales), se calcularon las medias, desviaciones típicas y se realizó un análisis de varianza (ANOVA) que puso de relieve los resultados que se presentan en la Tabla 1.

Tabla 1. Diferencias en autoconcepto, autoestima y sintomas psicopatológicos entro personas con y sin deficiencia auditiva

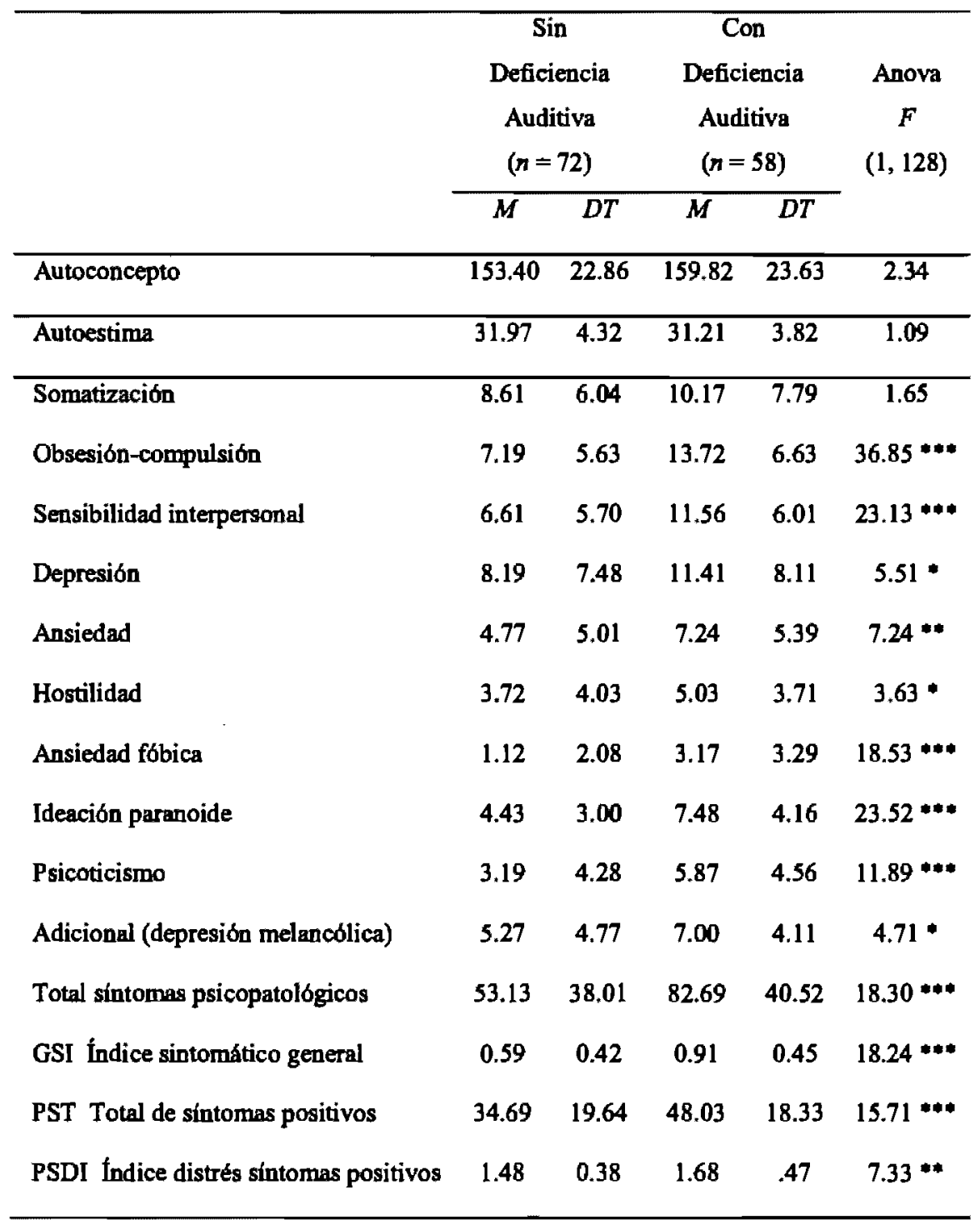

$" p<.05 * p<.01 * 0<.001$ 
Como se puede observar en la Tabla 1, en las variables autoconcepto y autoestima los sujetos con deficiencia auditiva no muestran puntuaciones diferencialmente significativas en relación a los sujetos normoyentes. Sin embargo, en síntomas psicopatológicos las personas con deficiencia auditiva tienen puntuaciones significativamente superiores en todas las escalas, excepto en la de somatización, es decir, se encuentra un mayor nivel de sintomas de obsesión-compulsión, de sensibilidad interpersonal, de depresión, de ansiedad, de hostilidad, de ansiedad fóbica, de ideación paranoide, de psicoticismo, de depresión melancólica, así como en el conjunto total de síntomas psicopatológicos, en el índice sintomático general, en el total de síntomas positivos, y en el indice de distrés por los sintomas positivos.

Autoconcepto, autoestima y síntomas psicopatológicos: Diferenclas de género en sujetos con y sin deficlencia auditiva

Con la finalidad de explorar la existencia de diferencias de género en el autoconcepto, la autoestima y los sintomas psicopatológicos en participantes con y sin deficiencia auditiva, se realizó un análisis de varianza (ANOVA). Los resultados obtenidos en las personas con deficiencia auditiva se presentan en la Tabla 2, y los obtenidos en normoyentes se exponen en la Tabla 3.

Como se puede observar en la Tabla 2 y 3, no se encontraron diferencias sigrificativas entre varones y mujeres ni en el autoconcepto, ni en la autoestima, $y$ apenas se encontraron diferencias en síntomas psicopatológicos. En los participantes con deficiencia auditiva únicamente se dieron puntuaciones significativamente superiores en las mujeres en el índice de sufrimiento por sintomas positivos, $F(1$, 56 ) $=4.51, p>.05$ (ver Tabla 2), y en la muestra de personas sin deficiencia auditiva, además de en el índice de sufrimiento, $F(1,70)=$ $6.23, p>.05$, también se encontraron diferencias significativas en la ansiedad fóbica, $F(1,70)=4.90, p>.05$, en ambos casos con puntuaciones superiores en las mujeres (ver Tabla 3 ). 
Tabla 2. Diferencias de género en autoconcepto, autoestima y síntomas psicopatológicos en personas con deficiencia auditiva

\begin{tabular}{|c|c|c|c|c|c|c|c|}
\hline & \multicolumn{2}{|c|}{$\begin{array}{c}\text { Total } \\
(n=58)\end{array}$} & \multicolumn{2}{|c|}{$\begin{array}{l}\text { Varmes } \\
(n=33)\end{array}$} & \multicolumn{2}{|c|}{$\begin{array}{l}\text { Mijeres } \\
(n=25)\end{array}$} & \multirow{2}{*}{$\begin{array}{c}\text { Anova } \\
F \\
(1,56)\end{array}$} \\
\hline & $M$ & $D T$ & $M$ & $D T$ & $M$ & $\overline{D T}$ & \\
\hline Antoconoepto & 159.82 & 23.63 & 161.63 & 22.20 & 157.44 & 25.67 & 0.44 \\
\hline Autoestima & 31.21 & 3.82 & 31.30 & 3.76 & 31.08 & 3.97 & 0.04 \\
\hline Somatización & 10.17 & 7.79 & 9.93 & 7.97 & 10.48 & 7.69 & 0.06 \\
\hline Obsesión-compulsión & 13.72 & 6.63 & 13.45 & 5.75 & 14.08 & 7.75 & 0.12 \\
\hline Sensibilidad interpersonal & 11.56 & 6.01 & 10.63 & 5.45 & 1280 & 6.58 & 1.86 \\
\hline Depresión & 11.41 & 8.11 & 9.75 & 7.15 & 13.60 & 8.89 & 3.32 \\
\hline Ansiedad & 7.24 & 5.39 & 6.54 & 5.17 & 8.16 & 5.65 & 1.27 \\
\hline Hostilidad & 5.03 & 3.71 & 4.27 & 3.18 & 6.04 & 4.17 & 334 \\
\hline Ansiodad fábica & 3.17 & 3.29 & 3.15 & 3.19 & 3.20 & 3.50 & 0.00 \\
\hline Ideación parancide & 7.48 & 4.16 & 6.90 & 4.16 & 824 & 4.11 & 1.46 \\
\hline Psicoticismo & 5.87 & 4.56 & 5.78 & 4.29 & 6.00 & 4.98 & 0.03 \\
\hline Adicianal (depresión melanoólica) & 7.00 & 4,11 & 7.30 & 3.99 & 6,60 & 4.32 & 0.41 \\
\hline Total síntomes psicopatológioos & 8269 & 40.52 & 77.75 & 37.09 & 89.20 & 44.58 & 1.13 \\
\hline GSI Índice sintamático genaral & 0.91 & 0.45 & 0.86 & 0.41 & 0.99 & 0.49 & 1.13 \\
\hline PST Total de sintomas positivos & 48.03 & 18.33 & 48.33 & 18.25 & 47.64 & 1881 & 0,02 \\
\hline PSDI Índice distrés sintomas pasitivos & 1.68 & 0.47 & 1.57 & 0.38 & 1.83 & 0.55 & $4.51 *$ \\
\hline
\end{tabular}

$* p<.05$ ** $p<.01$ *** $p<.001$ 
Tabla 3. Diferencias de género en autoconcepto, autoestima y sintomas psicopatológicos totales en una muestra de personas sin deficiencia auditiva

\begin{tabular}{|c|c|c|c|c|c|c|c|}
\hline & \multicolumn{2}{|c|}{$\begin{array}{c}\text { Total } \\
(n=72)\end{array}$} & \multicolumn{2}{|c|}{$\begin{array}{l}\text { Varcaes } \\
(n=40)\end{array}$} & \multicolumn{2}{|c|}{$\begin{array}{l}\text { Mijores } \\
(n=32)\end{array}$} & \multirow{2}{*}{$\begin{array}{c}\text { Anova } \\
F \\
(1,70)\end{array}$} \\
\hline & $M$ & $\overline{D T}$ & $M$ & $D T$ & $M$ & $\overline{D T}$ & \\
\hline Autocancepto & 153.40 & 2286 & 153.16 & 23.54 & 153.71 & 2234 & 0.0 \\
\hline Autoestima & 31.97 & 4.32 & 3230 & 4.22 & 31.56 & 4.47 & 0.51 \\
\hline Samatización & 8.61 & 6.04 & 7.60 & 5.35 & 9.87 & 6.69 & 257 \\
\hline Obsesión-compulsión & 7.19 & 5.63 & 7.25 & 5.79 & 7.12 & 5.51 & 0.00 \\
\hline Sensibilidad interpersconal & 6.61 & 5.70 & 6.07 & 4.82 & 7.28 & 6.65 & 0.79 \\
\hline Depresión & 8.19 & 7.48 & 727 & 5.78 & 934 & 9.14 & 1.36 \\
\hline Ansiedad & 4.77 & 5.01 & 4.55 & 4,40 & 5.06 & 5.74 & 0.18 \\
\hline Hostilidad & 3.72 & 4.03 & 3.62 & 3,62 & 3.84 & 4.56 & 0.05 \\
\hline Ansiectad fớbica & 1.12 & 208 & 0.65 & 1.59 & 1.71 & 247 & $4.90 *$ \\
\hline Ideación parancide & 4.43 & 3.00 & 4.45 & 278 & 4.40 & 3.31 & 0.00 \\
\hline Psicoticismo & 3.19 & 428 & 285 & 4.07 & 3.62 & 4.55 & 0.57 \\
\hline Adicional (depresión melanoólica) & 5.27 & 4.77 & 4.65 & 4.61 & 606 & 4.93 & 1.56 \\
\hline Total sintomas psioopatológioos & 53.13 & 38.01 & 4897 & 31.40 & 58.34 & 44.93 & 1.08 \\
\hline GSI Indice sintomático general & 0.59 & 0.42 & 0.54 & 0.34 & 0.64 & 0.49 & 1.08 \\
\hline PST Total de síntomas positivos & 34.69 & 19.64 & 34.62 & 19.00 & 34.78 & 20.72 & 0.00 \\
\hline PSDI f́ndice distrís sintumas positives & 1.48 & 0.38 & 1.38 & 0.35 & 1.60 & 0.40 & $6.23 *$ \\
\hline
\end{tabular}

${ }^{*} p<.05$ ** $p<.01$ *** $p<.001$ 
Relaclones del autoconcepto y la autoestima con sintomas psicopatológicos en personas con y sin deficlencia auditiva

Para analizar las relaciones del autoconcepto y la autoestima con diversos sintomas psicopatológicos en participantes con deficiencia auditiva y en sujetos normoyentes se calcularon coeficientes de correlación de Pearson cuyos resultados se presentan en la Tabla 4.

Tabla 4. Correlaciones entre del autoconcepto, y de la autoestima con sintomas psicopatológicos totales en una muestra de personas con y sin deficiencia auditiva

\begin{tabular}{|c|c|c|c|c|}
\hline & \multicolumn{2}{|c|}{$\begin{array}{c}\text { Sin } \\
\text { Deficiencia Auditiva } \\
(n=72)\end{array}$} & \multicolumn{2}{|c|}{$\begin{array}{c}\text { Con } \\
\text { Deficiencis Auditiva } \\
(n=58)\end{array}$} \\
\hline & $\begin{array}{c}\text { LAEA } \\
\text { Autoconcepto }\end{array}$ & $\begin{array}{c}\text { EA } \\
\text { Autoestima }\end{array}$ & $\begin{array}{c}\text { LAEA } \\
\text { Autoconcepto }\end{array}$ & $\begin{array}{c}\text { EA } \\
\text { Autoestima }\end{array}$ \\
\hline Somatización & -.12 & -.16 & -.10 & -.19 \\
\hline Obsesión-compulsićn & $-.34 *$ & $-.32 * *$ & -24 & -.18 \\
\hline Sensibilidad interpersonal & $-.31 *$ & $-.37+* *$ & -.25 & -.19 \\
\hline Depresión & $-.27 *$ & $-.35 *$ & $-.33 *$ & -.17 \\
\hline Ansiedad & -.12 & -.23 & -.07 & -.18 \\
\hline Hostilidad &.,$- 30 *$ & -.09 & -.07 & -.07 \\
\hline Ansiedad fobica & -.18 & $-26 *$ & $-28 *$ & $-35 *$ \\
\hline Ideación peranoide & -.17 & -.22 & -.13 & -.01 \\
\hline Psicoticismo & $-26 *$ & $-.22 *$ & $-.35 *$ & $-.40 *$ \\
\hline Adicional (depresión melancólica) & -.09 & -.17 & -.16 & -23 \\
\hline Total sintromas pricopatológicos & -27 & $-32 *$ & $-.27 *$ & $-26 *$ \\
\hline GSI Indice sintomático general & $-27 *$ & $-.32 \cdots$ & $-27 *$ & $-26^{*}$ \\
\hline PST Total de statomas pasitivos & $-27 *$ & $-.31+*$ & $-.40 \cdots$ & $-.38 *$ \\
\hline PSDI fodice distrits par sintomas positivos & -.01 & -.17 & -12 & -.07 \\
\hline
\end{tabular}

$\bullet p<.05 \quad \cdots p<.01 \quad \cdots p<.001$ 
Los coeficientes de correlación obtenidos (ver Tabla 4) ponen de relieve, tanto en la muestra de participantes con y sin deficiencia auditiva, la existencia de correlaciones significativas negativas del autoconcepto y la autoestima con síntomas de depresión, de psicoticismo, con la escala total de síntomas psicopatológicos, con el índice sintomático general y con el total de síntomas positivos. Complementariamente, en las personas con deficiencia auditiva se han hallado correlaciones significativas negativas del autoconcepto y la autoestima con síntomas de ansiedad fóbica, y en las personas normoyentes con sintomas de obsesión-compulsión y de sensibilidad interpersonal.

\section{DISCUSIÓN}

En primer lugar, los resultados obtenidos en este estudio muestran la ausencia de diferencias significativas entre personas con y sin deficiencia auditiva en el autoconcepto y en la autoestima. Estos resultados refutan la primera hipótesis que proponla que las personas con deficiencia auditiva tendrian puntuaciones significativamente inferiores. Aunque algunos estudios han puesto de relieve que los jóvenes y adultos sordos tienen niveles significativamente más bajos que la población general en autoconcepto (Weisel y Kamara, 2005) y autoestima (Bat-Chava, 1994; Mulcahy, 2002; Schlesinger, 2000), sin embargo los datos de este estudio ratifican los resultados de otras investigaciones que han señalado que las personas con deficiencias auditivas no necesariamente tienen un autoncocepto-autoestima más bajos que los sujetos normoyentes (Bat-Chava, 2000; Crowe, 2003; Emerton, 1996). Como señalan Jarnbor y Elliott (2005), existen diversos factores protectores que pueden favorecer el desarrollo apropiado del autoconcepto y la autoestima en las personas con discapacidad auditiva, tales como: a) un sistema comunicativo que desde edades tempranas facilite el establecimiento de vínculos adecuados a nivel familiar, así como su desarrollo cognitivo, afectivo y social; b) una modalidad de escolarización que de respuesta a las necesidades educativas especificas del sujeto con una deficiencia auditiva en particular, o c) la identificación con su grupo de referencia -la comunidad sorda-, que favorece la creación de un sentimiento de 
pertenencia que protege contra el impacto negativo del prejuicio y la discriminación.

En segundo lugar, los resultados obtenidos han puesto de relieve que las personas con deficiencia auditiva manifiestan puntuaciones significativamente superiores en la mayorla de los síntomas psicopatologicos respecto a las personas normoyentes, es decir, se han confirmado puntuaciones superiores en sintomas de obsesióncompulsión (conductas, pensamientos e impulsos que el sujeto considera absurdos e indeseados, que generan intensa angustia y que son dificiles de resistir, evitar o eliminar), de sensibilidad interpersonal (sentimientos de timidez y vergüenza, tendencia a sentirse inferior a los demás, hipersensibilidad a las opiniones y actitudes ajenas, incomodidad e inhibición en las relaciones interpersonales), de depresión (vivencias disfóricas, anhedonia, desesperanza, impotencia y falta de energía, ideas autodestructivas y otras manifestaciones cognitivas y somáticas caracteristicas de los estados depresivos) de ansiedad (manifestaciones clínicas de la ansiedad, tanto generalizada como aguda o «pánico», incluye signos generales de tension emocional y sus manifestaciones psicosomáticas), de hostilidad (pensamientos, sentimientos y conductas propios de estados de agresividad, ira, irritabilidad, rabia y resentimiento), de ansiedad fóbica (miedo persistente, irracional y desproporcionado a un animal o persona, lugar, objeto o situación, generalmente complicado por conductas de evitación o de huida), de ideación paranoide (suspicacia, centralismo autorreferencial e ideación delirante, hostilidad, grandiosidad, miedo a la pérdida de autonomia y necesidad de control), de psicotícismo (sentimientos de alienación social), de depresión melancólica (escala adicional), así como en el conjunto total de síntomas psicopatológicos, en el índice sintomático general (medida generalizada de la intensidad del sufrimiento psíquico y psicosomático global), en el total de síntomas positivos, y en el índice de distrés por los síntomas positivos... Excepto en los síntomas de somatización, en los que no se dieron diferencias, las personas con deficiencia auditiva manifiestan más síntomas psicopatológicos, con los que se ratifica la segunda hipótesis. Estos resultados apuntan en la misma dirección que otros estudios que han evidenciado que los jóvenes y adultos con deficiencia auditiva frecuentemente presentan 
puntuaciones más altas en sintomas psicopatológicos en comparación con sujetos normoyentes, especialmente síntomas ansiosos (De Graaf y Bijl, 2002; Fellinger et al., 2005; Tambs, 2004) y depresivos (De Graaf y Bijl, 2002; Mulcahy, 2002; Tambs, 2004).

En tercer lugar, los resultados de este estudio evidencian la ausencia de diferencias de género en el autoconcepto y la autoestima, tanto en los participantes con deficiencia auditiva como en las personas sin deficiencia, lo que confirma la tercera hipotesis. Estos datos apuntan en la misma dirección que otros trabajos realizados con jóvenes y adultos con sordera (Crowe, 2003), y también los encontrados en estudios con población general (Garaigordobil et al., 2005; Lameiras y Rodriguez, 2003; Matud et al., 2003).

En lo que a sintomas psicopatológicos se refiere, la literatura sobre la deficiencia auditiva ha encontrado diferencias significativas en función del género, con mayores niveles de sintomatologla en las mujeres (De Graaf y Bijl, 2002; Fellinger et al., 2005), en la misma línea que en la población normoyente (González de Rivera et al., 2002). Sin embargo, los resultados de este estudio apenas muestran diferencias de género en ambos grupos muestrales. En los participantes con deficiencia auditiva únicamente se dieron puntuaciones significativamente superiores en el índice de sufrimiento por sintomas positivos, y en la muestra de personas sin deficiencia auditiva, además de en este índice, se encontraron también diferencias significativas en la ansiedad fóbica, en ambos casos con puntuaciones superiores en las mujeres. Por consiguiente, se rechaza la cuarta hipótesis que planteaba que las mujeres con y sin deficiencia auditiva tendrlan puntuaciones superiores en síntomas psicopatologicos. Tal vez diferencias en las características muestrales de los distintos estudios puedan explicar estos resultados, ya que cuando se incorporan sujetos con niveles de estudios universitarios las diferencias de género en síntomas psicopatológicos se acentúan.

Finalmente, los coeficientes de correlación obtenidos sugieren que tanto en la muestra de participantes con y sin deficiencia auditiva, los que tenlan alto autoconcepto y autoestima informaron tener pocos sintomas de depresión, de psicoticismo, pocos sintomas psicopatológicos en el total de las escalas, bajo índice sintomático general, y pocos sintomas positivos. Complementariamente, las 
personas con deficiencia auditiva y alto autoconcepto y autoestima tenlan pocos sintomas de ansiedad fóbica, mientras que las personas sin deficiencia auditiva con alto autoconcepto y autoestima tenlan pocos sintomas de obsesión-compulsión y de sensibilidad interpersonal. Por consiguiente, se ratifica la quinta hipotesis del estudio que planteaba que los participantes con alto autoconcepto y autoestima tendrian pocos sintomas psicopatológicos. Estos datos apuntan en la misma dirección que otros estudios realizados con deficientes auditivos (De Graaf y Bijl, 2002; Satapathy y Singhal, 2001) y con población general (Fan y Fu, 2001; Montt y Chavez, 1996) ya que han hallado correlaciones positivas entre autoconcepto, autoestima y salud mental.

En términos generales los datos de este estudio ponen de relieve que los sujetos con deficiencia auditiva, en comparación con la población general, no evidencian diferencias significativas en autoconcepto-autoestima, lo que probablemente se relacione en buena medida con su identificación con la comunidad sorda (Bat Chava, 2000; Jambor y Elliott, 2005). En este sentido, cabe subrayar que más del $93 \%$ de los participantes con discapacidad auditiva eran usuarios habituales de la lengua de signos y que todos ellos perteneclan a asociaciones de sordos, lo que sin duda alguna puede considerarse indicador de un alto grado de identificación con su grupo de referencia.

Sin embargo, los resultados del estudio también permiten observar que la deficiencia auditiva es un factor de riesgo asociado a la presencia de diversos trastornos psicopatológicos, lo que tal vez podría explicarse en base a dificultades en el establecimiento de vínculos comunicativos tempranos (el $88 \%$ de los sujetos tiene padres oyentes), sobreprotección parental, malos resultados educativos, o dificultades laborales derivadas de su deficiencia (Muñoz, 2006). Por otra parte, como señala este autor, muchas de estas personas tienen una alta probabilidad de ser mal diagnosticados cuando acuden a los servicios de salud mental generales debido a problemas tales como: 1) dificultades en la comunicación entre el paciente y el profesional, los cuales no siempre pueden solucionarse mediante intérpretes oficiales y/o familiares; 2) desconocimiento de las caracteristicas propias de los sordos y de su comunidad (habitualmente, los profesionales no han tenido nunca contacto con personas sordas y desconocen algunas de sus caracteristicas diferenciales, considerando patológicas ciertas 
peculiaridades que no lo son en un contexto sordo); y 3) ausencia de instrumentos diagnósticos adecuados (utilizar para el diagnóstico de una persona sorda que padece un trastomo mental los mismos criterios que se emplean para una persona oyente es una práctica muy arriesgada dado que, en general, las pruebas psicométricas suelen ofrecer un mal perfil de las personas sordas).

Todo lo anterior pone de relieve la necesidad de proveer a nivel asistencial servicios adecuados para la valoración y tratamiento psicológico de este colectivo. Pese a que en la actualidad en España tan sólo existe una Unidad de Atención en Salud Mental para Personas Sordas (que se encuentra en el Hospital General Universitario Gregorio Marafión de Madrid), resulta evidente que este colectivo necesita de una asistencia especializada en el ámbito de la salud mental, con entornos fisicos en cuyo diseño arquitectónico prime la eliminación de barreras comunicativas para que resulten plenamente accesibles a las personas con discapacidad auditiva, y sobre todo, con profesionales especificamente capacitados para emplear los sistemas comunicativos más adecuados al paciente, principalmente la Lengua de Signos Española. De hecho cara al futuro, tal y como subraya Muñoz (2006), resultará imprescindible que más profesionales del ámbito de la salud se impliquen en la atención a este colectivo y que, paso a paso, más personas sordas se formen como profesionales de atención directa a los miembros de su propia comunidad.

\section{REFERENCIAS BIBLIOGRÁFICAS}

Allen, S., y Gilbert, P. (1995). A social comparison scale: Psychometric properties and relationship to psychopathology. Personality and Individual Differences, 19, 293-299.

Bat-Chava, Y. (1994). Group identification and self-esteem of deaf adults. Personality and Social Psychology Bulletin, 20(5), 494-502. Bat-Chava, Y. (2000). Diversity of deaf identities. American Annals of the Deaf, 145(5), 420-428.

Crowe, T. V. (2003). Self-esteem scores among deaf college students:

An examination of gender and parents' hearing status and signing ability. Journal of Deaf Studies and Deaf Education, 8(2), 199-206. De Graaf, R., y Bijl, R. V. (2002). Determinants of mental distress in 
adults with a severe auditory impairment: Differences between prelingual and postlingual deafness. Psychosomatic Medicine, 64(1), 61-70.

De las Cuevas, C., y González de Rivera, J. L. (1991). Perfil sintomático y diagnóstico en pacientes psiquiátricos ambulatorios. Psiquis, 12, 326-336.

Derogatis, L. R. (2002). SCL-90-R. Cuestionario de 90 sintomas revisado. Madrid: TEA. (trabajo original publicado en 1983).

Derogatis, L. R., Rickels, K., y Rock, A. F. (1976). The SCL-90 and the MMPI: A step in the validation of a new self-report scale. British Joumal of Psychiatry, 128, 280-289.

Derogatis, L. R., y Cleary, (1977). Confirmation of the Dimensional Structure of the SCL-90: A study in construct validation. Joumal of Clinical Psychology, 33, 981-989.

Emerton, R. G. (1996). Marginality, biculturalism, and social identity of deaf people. En I. Parasnis (Ed.), Cultural and language diversity and the deaf experience (pp. 136-145). Cambridge: Cambridge Unlversity Press.

Fan, F., y Fu, J. (2001). Self-concept and mental health of college students. Chinese Mental Health Joumal, 15 (2), 76-77.

Fellinger, J., Holzinger, D., Dobner, U., Gerich, J., Lehner, R., Lenz, G., et al. (2005). Mental distress and quality of life in a deaf population. Social Psychiatry and Psychiatric Epidemiology, 40(9), 737-742.

Garaigordobil, M. (en prensa). LAEA. Listado de adjetivos para la evaluación del autoconcepto en adolescentes y adultos. Madrid: Pirámide.

Garaigordobil, M., Durá, A., y Pérez, J. I. (2005). Síntomas psicopatológicos, problemas de conducta y autoconceptoautoestima: Un estudio con adolescentes de 14-17 años. Anuario de Psicología Clínica y de la Salud / Annuary of Clinical and Health Psychology, 1(53-63).

García, F., y Musitu, G. (1999). AF-5. Autoconcepto Forma 5. Madrid: TEA.

González de Rivera, J. L., De las Cuevas, C., Rodríguez Abuín, M., y Rodríguez Pulido, F. (2002). SCL-90-R, Symptom Cheklist 90 Revised, adaptación española. Madrid: TEA.

Jambor, E., y Elliott, M. (2005). Self-esteem and Coping Strategies 
among Deaf Students. Journal of Deaf Studies and Deaf Education, 10(1), 63-81.

Lameiras, M., y Rodríguez, Y. (2003). Age and sex differences in selfesteem among spanish adolescentes. Psychological Reports, 93, 876-878.

Lane, H. (1992). The mask of benevolence. New York: Vintage Books. McCarthy, J. D., y Hoge, D. R. (1982). Analysis of age effects in longitudinal study of adolescent self-esteem. Developmental Psychology, 18, 372-379.

Matud, M. P., Ibánez, I., Marrero, R., y Carballeira, M. (2003). Diferencias en autoestima en función del género. Análisis y Modificación de Conducta, 29(123), 51-78.

Montt, M. E., y Chavez, F. U. (1996) Autoestima y salud mental en los adolescentes / Self-esteem and mental health among adolescents. Salud Mental, 19 (3), 30-35.

Mulcahy, R. T. (2002). Cognitive self-appraisal of depression and selfconcept: Measurement alternatives for evaluating affective states. Dissertation Abstracts Intemational: Section B: The Sciences and Engineering, 62(10-B), 4796.

Muñoz, J. (2006, 16 de junio). Sordera y salud mental: La psicología frente a la deficiencia auditiva. Infocop Online. Recuperado el 19 de noviembre de 2006, de http://www.infocop.es/ view_article.asp?id $=879$ ycat $=5$

Santana, R., y Torres, S. (2003). Desarrollo comunicativo-lingüistico en el niño sordo profundo. En M. Puyuelo y J. A. Rondal (Eds.), Manual de desarrollo y alteraciones del lenguaje. Aspectos evolutivos y patología en el niño y el adulto (pp. 205-252). Barcelona: Masson.

Satapathy, S., y Singhal, S. (2001). Predicting social-emotional adjustment of the sensory impaired adolescents. Journal of Personality and Clinical Studies, 17(2), 85-93.

Schlesinger, H. S. (2000). A developmental model applied to problems of deafness. Journal of Deaf Studies and Deaf Education, 5, 349361.

Rosenberg, M. (1965). Society and the adolescent self-image. Princeton, NJ: Princeton University Press.

Silber, E., y Tippett, J. (1965). Self-esteem: clinical assessment and 
measurement validation. Psychological Reports, 16, 1017-1071. Swallow, S. W., y Kuiper, N. A. (1988). Social comparison and negative self evaluations: An application to depression. Clinical Psychology Review, 8, 55-76.

Tambs, K. (2004). Moderate effects of hearing loss on mental health and subjective well-being: Results from the Nord-Trondelag hearing loss study. Psychosomatic Medicine, 66(5), 776-782.

Watson, D. C. (1998). The relationship of self-esteem, locus of control, and dimensional models to personality disorders. Journal of Social Behavior and Personality, 13 (3), 399-420.

Weisel, A., y Kamara, A. (2005). Attachment and Individuation of Deaf/ Hard-of-Hearing and Hearing Young Adults. Journal of Deaf Studies and Deaf Education, 10(1), 51-62. 